

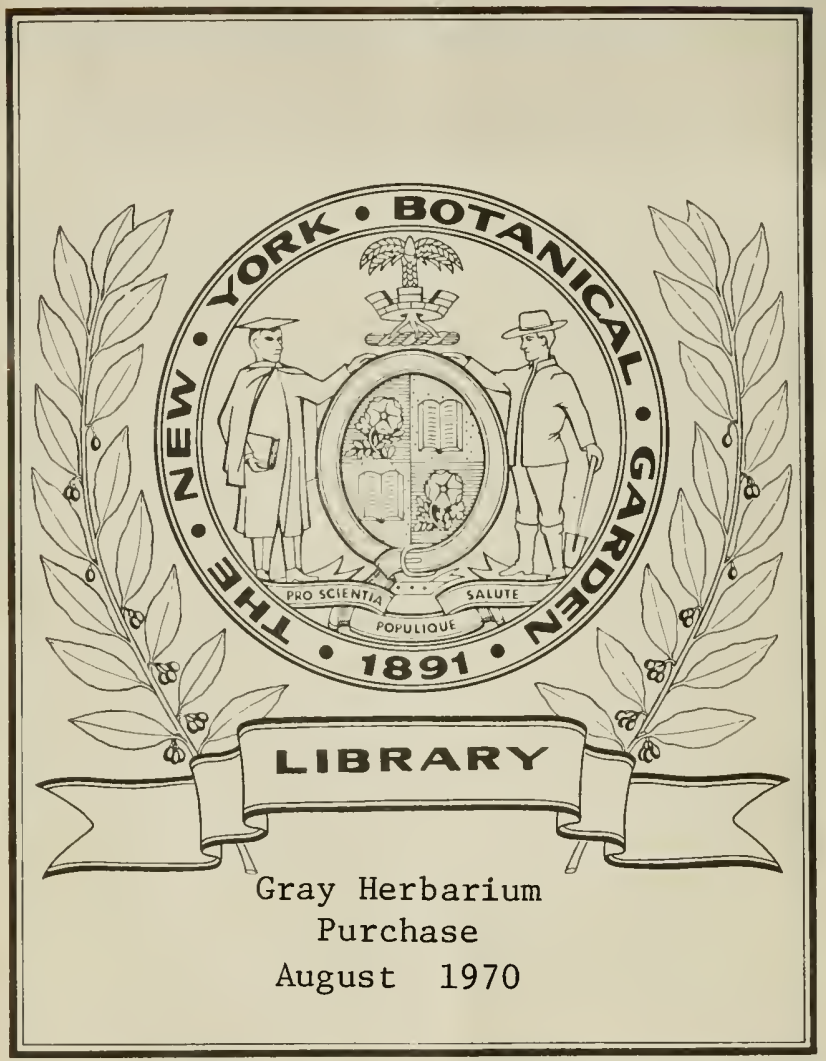




\section{FFDERICO KURTZ}

\section{DOS VIAJES BOTÁNICOS}

AL RIO SALADO SUPERIOR

(CORDILLERA DE MENDOZA)

EJECUTADOS EN LOS AÑos 1891-92 Y 1892-93

A rticulo publicado en el Boletix de la ACAdEmia Naciunal de Ciexcias DE Córdobs, tomo XIII, paiginces 171 y siquientes

\section{BUE.YOS AIRES}

IMPRENTA DE PABLO E. CONI É HIJOS, ESPECIAL PARA OBRAS

$$
680 \text { - C.alle Perú } \rightarrow 680
$$




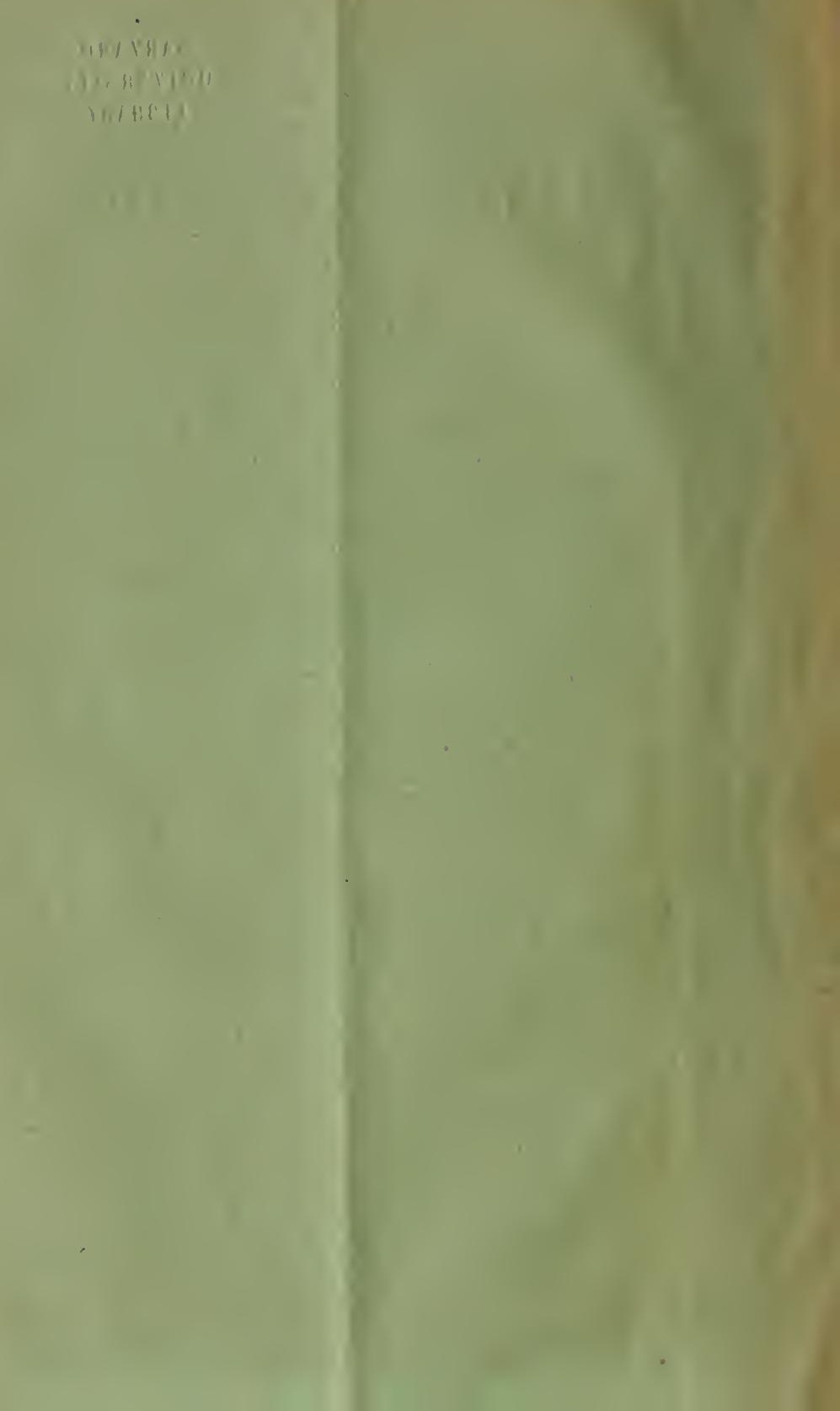




\section{FEDERICO KURT'}

\section{DOS VIAJES BOTÁNIGOS}

AL RIO SALADO SUPERIOR

(CORUILLERA DE HENDOZA)

EJECUTADOS EN LOS AÑOS 1891-92 Y 1892-93

A rtzculo publicalo en el Boletin de la Academa Nacional de Ciencias DE Cóndoba, tomo XIII, paginas 171 y siguientes

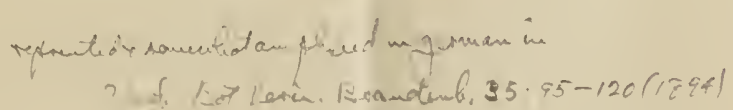

BUENOS AIRES

IMPRENTA DE PABLO E. CONI É HIJOS, ESPECIAL PARA OBRAS

680 - Calle perú - 680 


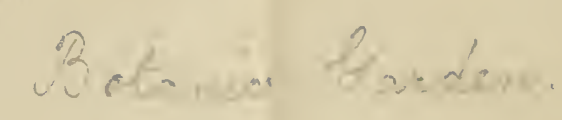




\section{VIAJES BOTÁNICOS AL RIO SALADO SUPERIOR}

(CORDILLERA DE MENDOZA)

E.IECUTADOS EX LUS AÑOS 1891-92 Y $1892-93$

POR FEDERICO KURTZ

La parte argentina de la Cordillera de los Andes, es decir, su falda oriental desde la frontera de Bolivia hasta el Estrecho de Magallanes, es indudablemenle la region más rica en plantas de toda la República, respecto al número de especies que componen su vegetacion, más rica aún que las provincias subtropicales.

Sin embargo, los conocimientos que se tienen sobre esta llora, son hasta ahora lejos de ser satisfactorios, circunstancia que tiene casi por única causa la escasez de observadores y coleccionistas y por tanto la de material de estudio. He aquí una ligera ojeada de lo que se ha escrito sobre el particular.

El primero que ha contribuido á la historia de las plantas audinas fué el médico inglés Dr. Gıllies, quien despues de una larga residencia en Mendozi, donde ejercía su profesion, volvió á Europa al fin de la segunda década de este siglo. Sus ricas colecciones, depositadas hoy en el Museo de KEw. cerca de Londres, han formado el objeto de una série de trabajos de W. J. Hoокer y G. WÁker Arrott, con impor- 
tantes contribuciones de su propia pluma $\left({ }^{1}\right)$. El Dr. Gillies formaba su herbario andino en la parte de la Cordillera comprendida entre el Paso de Uspallata al Norte y el valle del Rio Salado al Sur. A más de esta colección él ha herborizado tambien en otros puntos de la Provincia de Mendoza y en las de San Luis y Buenos Aires.

Despues de un intervalo de treinta años, empezaron á aparecer recien los trabajos del Dr. R. A. Philippi, Director del Museo Nacional de Chile, sobre plantas de la Cordillera entre Santiago de Chile y Mendoza y de las inmediaciones de esta ciudad, estudios que contienen la descripcion de muchas especies nuevas $\left({ }^{2}\right)$.

En 1871, Vicentio de Cesati, profesor de botánica en Roma, publicó descripciones y láminas de plantas que el naturalista Pellegrino Strober había encontrado en la bajada del Paso del Planchon tomando el camino de Mendoza $\left({ }^{3}\right)$.

Un discípulo de nuestra Universidad, el Dr. Salle EcheGaraY, formaba en 1875 un herbario de 291 especies en su Provincia natal, San Juan, en las cercanías de Leoncito y del Cerro Tontal, escribiendo el mismo una memoria sobre sus colecciones, de las que más tarde se ocupó detalladamente el Dr. J. Hienonymus $\left({ }^{4}\right)$.

El señor Gustavo Niederlein, que acompañaba al Dr. Pablo Lorextz en la expedicion del General Julio A. Roca al Rio Negro, volvió del Neuquen á Mendoza siguiendo el pié de la Cordillera, recogiendo en ese trayecto plantas que han sido descritas é incorporadas en el informe científico de dicha expedicion (i).

Más tarde, hace dos años, se publicó un artículo por J. BaLL dando noticias de las plantas que Mr. Williams Andrews

(1) Vẻase en el Apéndice I, $n^{\circ} 1$.

( $\left.{ }^{2}\right)$ Véase en el Apéndice $1, n^{0}$ ?.

$\left({ }^{3}\right)$ Véase en el $\Lambda$ péndice I, $n^{0} 3$.

(4) Véase en el Apéndice $I, n^{0} 4$.

$\left.{ }^{b}\right)$ Véase en el Apéndice I, n 5 . 
recogiera cerca del lago Lancar y otros puntos de la Patagonia ( $\left.{ }^{1}\right)$.

Finalmente, el autor de estas líneas ha escrito un informe de su primer viaje á la Cordillera tratando de la vegetacion que se observa entre Villa-Vicencio, Uspallata, Puente del Inca y Juncal en Chile ( $\left.{ }^{2}\right)$.

Estos son los trabajos que se ocupan directamente de la vegetacion de la falda argentina de la Cordillera, pues en la obra de Grisebach, Symbolae ad Floram argentinam, sólo se encuentran mencionadas plantas de las sierras de Catamarca, Silta y Jujuy; y de una espléndida coleccion de las sierras de Rioja, debida á los esfuerzos de los señores Hieron yuus y NieoERLEIN, clasificada ya en parte, no se ha publicado nada aún.

En el Museo Botánico de nuestra Universidad, el más rico en plantas argentinas que existe, la llora andina esta representada por las siguientes colecciones:

$1^{a}$ Un herbario regalado por el Dr. R. A. Philippi, que contiene una gran parte de las plantas descritas por el mismo en las obras mencionadas más abajo (Véase el Apéndice I, $\mathrm{n}^{0}$ 2);

$2^{\text {a }}$ El herbario del Dr. SaIle Echegaray, ilustrando los trabajos citados bajo el número 4 del Apendice I.;

$3^{\text {a }}$ Una cantidad de plantas de las cercanías del Lago Argentino, obsequio del teniente de navío señor CáriLos M. MoYano ;

$4^{a}$ Cuatro colecciones formadas por el infrascrito en varios viajes á la Cordillera, á saber :

a) Camino de Mendoza á Puente del Inca y Juncal en en Chile, 959 números;

b) Cerro Nevado, Cordillera entre el rio Salado al Norte y el lago Lancar al Sur, 1033 números;

c) Cordillera mendocina entre rio Salado y rio Malal-hué, 222 números;

(1) Véase en el Apéndice I, $n^{\circ} 6$.

${ }^{2}$ ) Véase en el Apéndice I, $n^{\circ} 7$. 
d) Camino de Mendoza á la Junta del Atuel y del Salado, Cordillera entre el Atuel, Salado y la frontera chilena, 280 números.

Samando todas estas contribuciones, me parece que tenemos representados en nuestro Yuseo como 1800 á 2000 especies de la flora andina, es decir, más ó menos la mitad de las que deben existir en esta region de la República Argentina. De Chile, que no es más que la falda occidental de la Cordillera con un litoral muy estrecho, en 1881 hemos conocido ya 53558 especies de plantas fanerógamas y criptógamas superiores ( 1 ).

Para dar ma idea de la riqueza de la flora de la Cordillera voy á mencionar el solo hecho de que durante nuestra estancia en el Cajon de Trolope, al pié del viejo volcan de Copá-hué, he recogido en las faldas del dicho volcan y en las inmediaciones de los baños termales y sulfurosos de Copáhué, 106 especies de plantas en dos escursiones.

Partimos para nuestro viaje de Villa Mercedes (Provincia de San Luis) donde se formaba la tropa y alistaba todo lo necesario para un viaje a través de la Pampa hasta San Rafael, hoy "25 de Iayo», nombre quese usa sólo oficialmente. El itinerario seguido para llegar á este último puutofué el siguiente: Villa Mercedes, Médanos Colorados, Chichaca Grande, Cerro Varela, Agua Dulce (Rio Salado), Paso de Tierra, Fortin Nuevo (sobre el rio Diamante) y entónces hemos seguido el rio Diamante hasta Rama Caida, que estí como í j leguas al E.-SE. de San Rafael. Despues de algunos dias de descanso emprendimos viaje en direccion al Cerro de la Guardia y de allí al Puente de Nihuil. Desde ese punto hemos seguido el rio Atuel arriba, hasta su confluencia con el Salado. Un poco más arriba estábamos en la Cordillera, siguiendo siempre el valle del rio Salado que hemos recorrido hasta su orígen.

(1) Vẻase en el Apéndice I, $n^{\bullet} 8$. 
M regreso visitamos Rodeo Viejo y Maial-huć, y de este establecimiento del General Ortega tomamos el camino á los Jagüeles (pequeíla sierra al Sur (lel Diamante), pasando para la junta del Atuel y el Salaulo, y para la Salina en la Pampa del Sur. 11 fin volviamos a tumir el camino de ida en Rama Caida para rolver á Córdoba.

Mi segundo viaje (Dicrembre de 1892 à Marzo de 1893), tenía por objeto la exploracion más detallada de la misma l'egion, es decir de la parte de la Cordillera entre los rios Atuel y Salado y el límite de Chile (Paso del Planchon). Sus resultados en la parte que se refiere a la Cordillera y sus contrafuertes están incluidos en este mismo informe.

De Villa Mercedes, con rumbo i los Médanos Colorados, se atravieza primero una zona de monte claro que sigue los bordes del Rio $5^{\circ}$ y cuya composicion me parece identica a la que se extiende al Sur de San Luis. Sus plantas más carac. terísticas son las siguientes :

Atamisquea emarginala Mrens. (Atamisque), en ejemplares arbóreos.

Bulnesia Retamo Griseb. (Retamo), en terrenosalgo salados.

Larrea divaricata GAv. (Jarilla hembra), aparece en esta region tambien bajo la forma de árboles elevados, elegantes.

Schinus dependens OrT. var. subintegra ExGL. (Duvaua praecox Grisen.; Molle), muy frecuente y muy variable en su tamaño y en la forma de sus hojas.

Gourliea decorticans GıL. (Chañar).

Prosopis alba Guseb. (Algarrobo).

Prosopis Algarrobilla Griseb. (Caiden), árbol bajo muy frecuente.

Aspidosperma Quebracho blanco Schldo. (Quebracho blanco), árboles altos generalmente aislados.

Jodina rhombifolia Hook. et Ars. (Arbol peje; en la Provincia de Córdoba : Quebracho flojo).

Verbesina encelioides (C.v.) Вехтн. et Ноок, muchas 
veces se observa donde hay hacienda vacuna, formando círculos bajo los árboles (Algar'obos) que los animales buscan por la sombra.

Hyalis argentea Dov. (Blanquillo), en terrenos secos, arenosos, formando á veces matas extensas.

Justicia campestris Griseb. (Quiebra-arado; en la Provincia de Córdoba tiene el mismo nombre la Heimia salicifolia Lk. et OтTо); muy frecuente.

Aristida sp. (Asaitillo); cubre muchas veces grandes espacios y se dice que es un buen pasto; no se le observa al Sur del Dianmute.

Un poco antes de llegar a los Médanos Colorados empieza la region de los Médanos, uno de los paisajes más tristes que conozco y que con algunas interrupciones se extiende liasta la confluencia del rio Salado con el Diamante, aunque variando algo el carácter de su vegetacion segun la cantidad de agua que está en el suelo ó en sus alrededores (en las cercanías de los grandes rios). El mismo carácter, monótono y depresivo en su vegetacion, presenta el vasto espacio de la Pampa comprendido entre el Cerro Varela (al Sud de San Luis) al Este, el pié de la Cordillera al Poniente, el Rio Diamante al Norte y el límite setentrional de la flora patiagónica (situado más ó menus en los bordes del rio Agrio, cerca de N̂orquin), al Sur. Toda esta inmensa region pertenece á la misma formacion botánica á excepcion de algunos puntos que salen como isletas en este océano pampeano, v. gr. el Cerro Varela, el de la Guardia y algunos grupos de médanos.

\section{LA PAMPA}

La Pampa, tal como se presenta entre los límites arriba indicados, se divide muy naturalmente en las siguientes subformaciones : 
1) La Pampa llana, y $1^{\text {a }}$ los Salitrales ;

2) Los Médanos, cimas de arena movedisa hasta de 20 metros de altura;

3) Los valles de los rios y de las lagunas ;

4) Las sierras pampeanas.

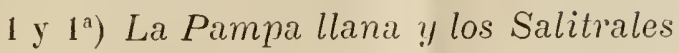

La Pampa se presenta generalmente como una planicie más ó menos ondulada ó completamente llana, de colores claros (banquizco, pampa de gramas, especialmente de Andropogoneas, Tupe (Panicum patagonicum Hreros.) y Junquillo (Diachyrium arundinaceum Griser.), verde-gris ó gris (pampa de Chenopodiáceas ó tambien salitrales), interrumpida ó punteada en algunas partes por manchas ó líneas negras : grupos de monte, que cuando afectan la forma lineal señalan el curso de algun arroyo ó una hajada un poco húmeda. Los arbustos más frecuentes en la Pampa son : Molle en sus varias formas (Schinus dependens, ORT. var. subintegra ExGr.) y Solupe (Ephedra americana IV. y E. ochreata Miers.). Muy raras veces se nota un árbol de un color verde claro, el Sauce hediondo (Salix Humboldtiana W.) ó la elevada forma del álamo de Italia (Populus pyramidalis Roz.), indicio seguro de agua ó de una habitacion cualquiera. Es característico para la Pampa, y puede observarse en cualquiera de sus partes, el predominio de una ú otra planta, de tal modo que puede hablarse de Cachiyuyu-pampa, Zambapampa, Tupe-pampa, Junquillo-pampa, Verbena-pampa ( Verbena seriphioides GrLL. et Ноок. y Lippia foliolosa PHL.), Chinita-pampa (Grindelia speciosa BTH.), Blanquillo-pampa (Hyalis argentea Dox.).

Entre la Pampa y los Salitrales que se encuentran en la region de que tratamos, los límites no pueden determinarse 
de un modo claro y preciso, pues sucede que hay un número de plantas halófitas que aparecen tambien en la Pampa (Zamba, Cachiyuyu, Brizopyrum, Juncus acutus LAм.) mientras el Chañar no parece ser suceptible á resistir mucha sal : Io he encontrado muchas veces en la region intermedia entre la Pampa y los Salitrales. Plantas que viven solamente en terrenos salados sou, por ejemplo: Jume (Suaeda divaricata Moce.) y además Spirostachys patagonica Griseb. y S. vaginata Griseb., Halopeplis Gilliesii Griseb., Niederleinia juniperoides HIEr., Statice brasiliensis Borss., Cortesia cuneata R. et P., Lippia salsa Griseb., Prosopis strombulifera Втн. De todas estas plantas es la más notable y singular la Niederleinia juniperoides, pequeño arbusto muy frágil, de un gris verde, que se asemeja mucho en su aspecto á ciertas formas del género. Juniperus y en especial á la que se cultiva en los jardines botánicos bajo el nombre de J.prostrata Pers. (J.horizontalis Мrch.). Como lo ha mostrado J. Heromyues (Bol. de la Acad. Nac. de Ciencias, t. III, 1879, p. 219-30 con lím.), pertenece esta planta á la pequeña familia de las Frankeniaceas, conocida hasta entónces solamente de la region mediterránea y de la isla de Santa Helena. La Niederleinia fué descubierta por G. Niedercers, durante la expedicion del Cieneral Roca al Rio Negro, en las orillas de los pantanos salados "Narraco ", poco al Norte del rio Colorado, y el que estas líneas escribe la ha encontrado desde Tendrica (al pié del Cerro Nevado) y la Laguna Llancanelo al Este. hasta la junta del Atuel y Salado, y Agua Nueva al Oeste y Norte, formando céspedes bajos de hasta un metro de diámetro que cubren á veces grandes espacios de terreno. 
2) Los Médanos

Cuando los médanos se presentan con el carácter de travesía, como por ejemplo entre Médano Colorado y Chichaca Grande, carecen completameute de árboles, apenas se observa de vez en cuando un pequeño Chañar estropeado, siendo la única planta leñosa de alguna frecuencia una clase de Solupe (Ephedra ochreata Mrens), que forma grupos circulares. Por lo demás, la regetacion se compone de algunas gramas (Andropogonis spec. 2), que se encuentran tambien en Ia Pampa baja, y de algunas Compuestas y Verbenaceas (Hyalis argentea Dox., Baccharis axillaris Dox.: muy frecuente, Trichocline sp., Lippia salsa Gr., Verbena sp.).

Sin embar'go, son generalmente los médanos los portadores de los grupos ó islas de montes que se observan en la Pampa y que en las cercaníns de los rios se presentan á reces en forma de bosques más ó ménos grandes. Los elementos de estos grupos aislados de monte son los mismos en toda la region, hélos aqui :

Atamisquea emarginata Miers. (muchas veces arbórea).

Tricomaria Usillo Ноок. (Csillo).

Larrea divaricata $\mathbf{C A V}_{\mathbf{A}}$.

Larrea cuneata CAV. (Jarilla crespa); encontrada por primera vez en el Diamante frente á San Rafael, pero que se Lalla tambien, mucho más al Oriente, en San Luis, Córdoba, Catamarca.

Bulnesia Retamo Griseb. (Retamo).

Condalia lineata A. Gray (Piquillin), raro.

Schinus dependens OrT. (Molle).

Gourliea decorticans GiLL.

Acacia striata Bтн. (?, Pichana de perro). 
Cyclolepis genistoides Dor., en su aspecto muy semejante al Usillo.

Chuquiraga erinacea Dor.

Lycium sp.

Tricycla spinosa Cav. (Monte negro); más cerca de la Cordillera.

Ephedra ochreata Miers. (Solupe).

Hyalis argentea Dos.

Lippia foliolosa PHuL. (Tomillo hembra).

Panicum patagonicum Hieros. (Tupe).

Diachyrium arundinaceum Griseb. (Junquillo).

En forma de árboles se presentan sólo tres: Chañar, Calden y Retamo, encontrándose á veces Molle y Atamisque en ejemplares de dos y más metros de altura pero nunca con tronco bien desarrollado. El Chuquiraga (Chuquiraga erinacea Don.) que se cría con frecuencia en los médanos es uno de los arbustos más feos, espinosos, y desagradables que pueden imaginarse, siendo las brácteas del involucro de sus flores mismas transformadas en espinas.

3) Las orillas de los rios y de las lagunas

Las márgenes de los rios Diamante y Atuel son los que se han podido estudiar más detalladamente. Este último, como el rio Salado que separa las provincias de Mendoza y San Luis, es un rio pampeano típico, es decir, carece de una formacion litoral bien definida y la Pampa se acerca hasta el rio mismo sin que medie una zona de monte como, por ejemplo, la que acompaña al rio Diamante á lo menos en su márgen derecha.

Las plantás que constituyen la flora litoral del rio Diamante son muy abundantes en número aunque de muy pocas especies, formando cada una zonas extensas, donde una misma especie cubre muchas cuadras y hasta media legua, 
las que llevan los nombres de Cortaderales, Pichanales, etc. naturalmente bastante monótonas.

Las principales entre estas plantas son cuatro:

Gynerium argenteum Nees. (Cortadera).

Heterothalamus sparlioides Hook. et Ars. (Pichana).

Baccharis salicifolia Pers. (Chilca).

Juncus acutus Las. (Junco).

La Cortadera se cría generalmente á la orilla del rio y en los pantanos inmediatos, formando lindísimos grupos que constitnyen una zona de un blanco argentino que desde lejos indica la direccion del rio.

Un poco más distante de este se encuentran la Pichana y la Chilca, con una altura que varía entre $0.3 \mathrm{~m}$. y 2 a $2.5 \mathrm{~m}$. (Chilca).

El Juncus se encuentra con menos frecuencia que las otras, prefiriendo terrenos algo salados, y forma con sus hojas rígidas y punzantes grupos de un verde oscuro.

Como se ha dicho ya, estas plantas, por lo general, se presentan solas una al lado de la otra, pero á menudo se hallan mezcladas, especialmente la Pichana y la Chilca.

Entre las yerbas que se encuentran en la margen del rio las más características son : Pluchea Quitoc DC., Glycirrhiza astragalina GıL., Prosopis strombulifera Втн., Distichlis sp. 2, etc.

El único árbol que existe cerca del rio es el Chañar, el que se presenta algunas veces en ejemplares grandes. Por el contrario, los médanos que acompanan al rio Diamante por su márgen derecha, son cubiertos continuamente de un monte denso compuesto de los mismos elementos que caracterizan esa formacion y además de: Prosopis siliquastrum DC., Prosopis Algarrobilla Griser., Adesmia trijuga Gill., Proustia ilicifolia H. et A., Doniophytum anomalum (Dox.) M., Sclerophylax Cynocrambe Griseb., Verbena ceratophylla GıL. et H., Verbena flava GiLs. et H., Munroa squarrosa Torr. (Munroa mendocina Puı.). 
Las orillas del rio Atuel en su parte pampeana no presen tan una llora litoral y rada indica su rumbo á no ser algunos cortaderales. Por lo general, la Pampa, cubierta de Tupe, Junquillo ó Zamba, llega hasta la márgen misma del rio, pero más arriba de su confluencia con el Salado aparecen en el aluvion algunos tipos serranos como Adesmia trijuga Gilt., Senecio salsus Gruseb.

Por falta de tiempo, muy pocose ha podido estudiar la flora de la Laguna Llancanelo situada al pić de la falda occidental del Cerro Nevado, la que cerca de la desembocadura del rio Malal-hué tiene orillas pantanosas cubiertas de densos totorales, encontrándose al lado de la Totora (Typha domingensis Pers.) una especie de Phragmites (P. dioica Hǿк..). (Los totorales más grandes que he visto en la República son los que adoruán las orillas del Curú-leubú, cerca de Chushmalal). A poca distancia de la laguna tenemos un monte claro (Molle pispo, Alpataco, Piquillín, Montenegro etc.), que cubre tambien el Cerro de los reones. En algunos lugares algo salados se veían Statice brasiliensis Boıss. y Niederieinia juniperoides Heron., dominando el Junquillo en el campo vecino á Malal-hué.

\section{4) Las sierras pampeanas}

Bajo esta denominacion pueden comprenderse el Cerro Varela al sud ó sudeste de San Luis y el Cerro de la Guardia al sud de San Rafael, el que como toda la sierra de San Rafael, de la que forma parte, no es sinó el borde de la altiplanicie que se extiende entre el Cerro Nevado y la CordiLlera, limitada al norte por los ríos Atuel y Diamante (segun el Dr. G. Bodenbender).

El Cerro Varela que se presenta como una pequeña ca- 
dena de montanas dirigida de norte á sud y compuesta de pórlido cuaréfero, en su mayor parte colorado, posee cerca del Puesto Varela, en su pendiente oriental, contornos muy pintorescos y valles circulares, pero la falta de corrientes de agua hace que la vegetacion sea muy escasa, y su monte demasiado claro, se compone de los tipos mas simples de los médanos, como, por ejemplo, Larrea divaricata Cav. Entre las pocas plantas que he observado merecen mencion las siguientes:

Pitcairnia spathacea Gusen. (frecuente).

Monnina linearifolia R. et P.

Hyaloseris cinerea Gr. var. tomentella Gr.

Salvia Gilliesii Bтh. var. Lorentzii (Gr.) Henor.

El Cerro de la Guardia, pequeño grupo aislado al sur de San Rafael, presenta la misma flora que las sierras airidas frente á la cordillera (Sierra de Tunuyau) caracterizada por algunos tipos sub-andinos que se hallan junto al monte pampeano, el que entra y sube siguiendo los valles de los arroyos. Así aparecen en el Cerro de la Guardia los siguientes tipos: Larrea nitida CAv., Cereus sp. y Gochnatia glutinosa Don.

Poco abajo de la cumbre, en las cercanías de la Fuente de la Guardia, se divisan las dos plantas que tanto predominan en las riberas del Diamante: la Cortadera y la Pichana, aunque ejemplares algo bajos.

La vegetacion de los valles comprende, á más de las especies ya varias veces mencionadas, como Atamisque, Retamo, Piquillín, Molle, Calden, Chañar, Montenegro, las siguientes:

Larrea nitida Cav. (Jarilla de la sierra), raro.

Caesalpinia praecox R. et P. (Brea), frecuente en los valles y notable por sus ramos verdes. Se presenta aquí en forma de sub-arbusto, sin tronco principal, pero á juzgar por las hojas y frutas es indudablemente idéntica con la Caesalpinia praecox de San Luis y Córdoba. 
Poinciana Gilliesii Hook., pequeño arbusto hasta de 1 metro de altura.

Cassia aphylla Cav., en una forma muy baja teniendo à lo más 1.5 a 3 decímetros de altura.

Zuccagnia punctata CAv. (Jarilla macho), frecuente en los bordes de los arroyos, presenta en su aspecto mucha semejanza con las verdaderas Jarillas, especialmente con Ia Larrea divaricata CAv.

Cereus sp. (véase el texto más abajo).

Gochnatia glutinosa Don., arbusto que con sus pequeñas hojas relucientes juntas con el pappus de las cabezuelas florales, recuerda algo el Myrtus communis $\mathbf{L}$.

Proustia ilicifolia H. et A. (Altepe), abundante en los arroyos secos.

Mikania tenuiflora Gruseb., enredadera muy frecuente en lugares algo húmedos.

Chenopodium papulosum МocQ. TAxD., raro en terrenos arenosos.

La planta más característica de esta seca region es el Cereus (con flores coloradas pequeñas muy numerosas) que en forma de columnas verticales de 0.5 hasta $1.5 \mathrm{~m}$. de altura y 0.2 á $0.3 \mathrm{~m}$. de diámetro adorna de un modo extraño las lomas superiores, semejando á lo lejos cristales gigantescos ó columnas basálticas.

\section{LA CORDILLERA}

1) La region sub-andina

Siguiendo el río Salado, aguas arribas, desde su confluencia con el Atuel, se encuentra cerca de su desembocadura ó salída de la sierra entre las primeras colinas y lomitas de la cordillera, una estrecha zona que hemos podido estudiar desde 
San Cárlos (hoy "9 de Julio») al norte, hasta más al sur de Malal-hué y que está ocupada por una vegetacion que combina elementos del Monte panpeano (Holle, Jarilla, Piquillin, AIgarrobo) con cierto número de tipos liasta alora no observados ú observados en muy escasos ejemplares y que llamamos subandinos. Como tales pueden considerarse Ephedra ochreata Miers. (Solupe), Verbena aphylla GuL. et Ноoк., Tricycla spinosa Cav. (Montenegro), Larrea niticla Car., Colliguaya integerrima GiLL. et Hоoк. (Colliguay), Cassia conjugata R. et P., y el Chacay (Colletia Doniana Cuos.); el Chacay es un lindo árbol, el último en la Cordillera que acompaña los arroyos, generalmente en union con la Colliguayja integerrima Gı́. et Ноок., arbusto de ramaje verde claro brillante; más arriba todas las plantas leñosas: Molle, Leña Amarilla, Anarthrophyllum, se presentan en forma de arbustos que no alcanzan más que hasta 2.5 m. de altura.

Estas especies sub-andiuas aparecen de repente en las faldas de la Cordillera, único punto donde existen, para desaparecer muy luego un roco más arriba. El Chacay se halla, aunque en forma de pequeño arbusto, hasta Alverjalitos; la Ephedra ochreata Mreis. cede pronto á las varias formas de la E. americana W., planta muy comun en el valle del río Salado, y Tricycla, con Cassia conjugata R. et P. y la Colliguaya, las plantas mis características de la zona sub-andina, apenas si entran en la Cordillera. De las plantas más genuinas del monte pampeano desaparecen al pié de la Cordillera : el Chañar, Larrea divaricata CAv., L. cuneata CAv., los Algarrobos, la Chuquiraga crinacea Dox; mientras que el Piquillín se observa hasta más arriba de Los Molles y el Molle sube más ó menos hasta el límite de los arbustos, junto con la Leña Amarilla (Aclesmia pinifolia Grir..), pero parece no pasa la cumbre, por lo menos no recuerdo haberlo encontrado al otro lado en el Valle Hermoso. 
Al lado de los tipos locales sub-andinos se divisan algunas especies andinas, como ser la Adesmia trijuga GrL. var. robusta Ноок., y la Dipyrena glaberrima Gict. et Ноок.

Las plantas encontradas en esta zona intermedia entre la Pampa y la Cordillera se hallau en la lista siguiente :

Plantas de la region sub-andina

Monnina dictyocarpa Gurseb.- Embocadura de los ríos Salado y Atuel.

Larrea nítida Gav. - Arroyo Chacay.

Drosopis Alpataco PHrL. (?) - Agua caliente; arbusto de 1 a $1.5 \mathrm{~m}$ de altura, formando bosques extensos.

Cassia conjugata R. et P. - Cieneguita; arriba de la embocadura del Atuel en abundancia.

Anarthropleyllun rigidum (GıL.) Вехтн. et Ноок. Cerro de las Leñas Amarillas (la verdadera Leña amarilla, Adesmia pinifolia GrLt., no se cría á tan baja altura).

Colletia Doniana Cros. (Chacay). - Arroyos Faja, Manzanas, Chacay, Pequenco, La Cieneguita.

Gochnatia glutinosa Don. - Barranca del río Diamante, etc.

Proustia ilicifolia Hook, et Ars. (Altepe).- Arroyo Fa ja; arbolito hasta de $3 \mathrm{~m}$. de altura.

Grindeliaspeciosa Benth. (Chinita).-Desde Yaucha hasta la Sierra de Loncoche y más al sur; una de las plantas más frecuentes y muchas veces predominante.

Gutierrezia spathulata (Pнu.)m., var. cochroleuca m. - Entre los arroyos Papagayos y Houdo muy frecuente. La planta típica de la Cordillera tiene flores amarillas; la variedad las tiene blancas y algo amarillentas.

Śnecio albicaulis Hook. et Arx.- Muy frecuente.

S. salsus Guseb.-Yaucha, Bajada al Papagayos. 
Chuquiraga erinacea Don (Cola de zorro). - Entre Diamante y Faja, Coy-hueco; frecuente.

Doniophytum anomalum (Dox) m. - Embocadura del Salado.

Lippia foliolosa PHuc. (Tomillo hembra).-Desde Yancha hasta Malal-hué; frecuente.

Verbena aphylla GicL. et Ноок. - La Cortadera hasta el río Salado, algunas veces en forma de grandes bosques, es el arbusto predominante. Parece que existe otra especie muy semejante mis robusta y más alta, pero de ella (observada especialmente entre el Cerro Mesa y Malal-hué) no tengo materiales suficientes para una determinaciou.

$V$. flava Gicl. et Hook.-Arroyo Papagayos, frecuente; tiene flores amarillas ó blanco-violáceas.

$V$. glauca Gıь. et Ноок.-Arrgyo Carrizalito.

$V$. seriphioides GıL. et Ноок. (Tomillo macho).-Desde Yaucha al sur muy frecuente.

Salvia Colliesii Bru.-Arroyo Faja; arbustos lasta 2 m. de altura.

Plantago patagonica JACQ. - La Cortadera.

Tricycla spinosa Cav. (Montenegro). - Arroyo Hondo, Malal-hué; frecuente.-Al sur hasta el Río Negro (J. Hierowrus, Sertum patagonicum, No 24).

Colliguaya integerrima Gics. et Ноок. (Colliguay).La Cieneguita hasta el Portezuelo del mismo nombre, en abundancia ; en otras partes de esta region no la he visto; pero la conozco tambien de Villa-Vicencio, idonde se cría bajo condiciones semejantes.

Ephedra ochreata Mrars. (Solupe). - Coy-hueco, Embocadura del río Salado, La Cieneguita, frecuente. Las frutas, de un color rojo-claro, dulces, comestibles, son las más grandes de las especies argentinas del género Ephedra.

Gynerium argenteum Ners. - Pantano de la Cieneguita (ejemplares muy pequeños); Arroyo de la Cieneguita y más arriba hasta Los Morros. 
Stipa Neaci Nees. - Embocadura del Salado, La Cieneguita; planta muy linda.

En el valle del arroyo Cieneguita, más ó menos en el límite de la zona sub-andina, existe un pantano que presenta especies de Ranunculus, Anagallis, Mimulus, Plantago, Armeria, Juncus, Scirpus, Koeleria, y los ejemplares más pequeños de Gynerium que jamás he visto: toda la planta no tenía más de 15 á 30 centímetros del altura. Este pantano estaba encerrado por bosques de Chacay y Colliguay, divisándose en las pendientes secas los brillantes racimos de la Cassia conjugata R. et $\mathbf{P}$., y más arriba los espinosísimos grupos del Crucero (Berberis Grevilleana Gict.) entrelazados por la Mutisia retrorsa Cav., enredadera muy frágil con grandes flores amarillas, que segun he observado parece buscar siempre la proteccion de cualquier arbusto espinoso como lo hace tanbien otra linda planta: la Stipa Neaei Nees.

Como límite superior de la zona sub-andina se puede fijar, muy naturalmente, la aparicion ó límite inferior de la Leña Amarilla (Adesmia pinifolia GrLu.) con tanta más razon, cuanto que al mismo tiempo que este arbusto aparece una série de otras plantas hasta entónces uo observadas.

Lu poco más arriba, siguiendo el arroyo de la Cieneguita se atraviesa el Portezuelo de la Cieneguita que divide el vallle de este nombre de el del Rio Salado. En la cima de este Portezuelo y en su falda al Salado encontramos por primera vez un mímero de plantas que indican el principio de una nueva region. Estas son: Adesmia pinifolia, A. lrijuga y Anarthrophyllum rigidum, formando bosques junto con Ephedra americana, Tetraglochin strictum y Cassia $A r^{\prime-}$ nottiana. De plantas bajas, matas y yerbas, se observan: Malesherbia solanoides, Argylia Bustillosii, Schizanthuis sp., Acaenasp., Nassauria axillaris, presentandose esta última en forma de corales blanquizcos. Entre las plan- 
tas leñosas mencionadas se presenta, como la más característica y comun, al par que sirviendo para marcar el límite superior de la vegetacion leñosa de esta region, la Leña amarilla. Esta circunstancia y la de criarse en el reino de la Adesmia pinifolia una llora bien caracterizada me han inducido a llamar esta zona: la region de la Leña amarilla ó region andina media, con sus límites bien sencillos: desde donde aparece, hasta donde deja de existir la Leña amarilla.

\section{2) La region andina mədia}

(Region de la Leña amarilla)

Desde la cima del Portezuelo de la Cieneguita se divisa la parte superior del valle del rio Salado bastante ancho, con rumbo general de Este al Oeste y rodeado de considerables sierras de pendientes rápidas, cuyas cimas están en algunas cubiertas de nieve, como por ejemplo la Sierra de los IIolles, los Cerros de los Blancos y de los Morros y la Sierra del Portezuelo ancho. Al frente de la pequeña laguna de las Piedras Negras, pasa el Salado por una pequeña garganta, pero un poco más arriba los cerros de su márgen izquierda se retiran al Norte para formar así el pintoresco valle de los $110-$ lles. Arriba de Los Molles, en el valle del Salado, conserva un cierto ancho hasta poco arriba de los Morros, estrechándose luego cada rez más hasta la Cuesta del Portezuelo ancho.

A los dos lados del valle principal del rio Salado desenbocan otros valles pintorescos: del lado derecho los de las Vayas altas, de las Ánimas, de las Aguas amarillas, de los Morros y de la Yesera; del izquierdo, el valle de los Molles, el cajon de Ubilla y el valle del Alverjalito.

Con respecto á su vegetacion, el valle del rio Salado, como casi todos los de la Cordillera alta, ofrece á primera vista un 
aspecto más ó menos desnudo, no se nota en parte alguna una capa de plantas contínua de alguna extension; en el fondo del valle y en ciertas alturas se observan bosques ralos formados siempre de los mismos elementos, que se suceden, segun su grado de frecuencia, en el siguiente órden: Molle, Adesmia trijuga, Chuquiraga oppositifolia, Dipyrena, Ephedra, Cassia, Berberis Grevilleana, Anarthrophyllum, Tetraglochin; y de plantas más bajas: Argylia, Verbena spathulata, Nassauvia axillaris. Wás arriba, en la sierra, se presentan grupos de Leña amarilla, Molle y Dipyrena, y al fin queda solamente la Leña amarilla formando grupos aislados que, vistos de abajo, se destacan como manchas negras del color moreno de las rocas.

Prados, ó algo semejante, hay cerca de Los Molles y en el suelo de algunos valles, pero ellos están formados por plantas introducidas al mismo tiempo que la hacienda vacuna como por ejemplo la Verbesina encelioides [CAv.] Bтн. et Поок., Astragalus unifultus L'Her., Glycirrhiza astragalina GiLL.

Las pequeñas manchas verdes que de vez en cuando se observan en las pendientes de los cerros, son pantanos ó cićnegas de poca extension, y la vegretacion se limita, en general, á estos y á las márgenes de los arroyos.

Al lado de esta vegetacion, algo contínua y característica, tenemos un número de plantas que se crían en grupos aislados ó en pequeñas comunidades en el suelo del valle y las cercanías de las pendientes, pudiendo mencionarse entre ellas las siguientes: Malesherbia, Mulinum spinosum, Azorella Gilliesii, Rhodolivion, Melosperma, Tropacolum polyphyllum, Lathyrus macropus, y dos ó tres especies de Calicéreas.

Entre las plantas que acompañan al agua en sus varias formas (véase la lista más abajo), se encuentra como especie notable Triglochin maritima $\mathbf{L}$. que se balla en abundancia en el barro en parte caliente y fuertemente sulfuroso de los 
Baños de los Iolles, en union con Distichlis thalassica (Ктн.) Desv., Juncus sp., Baccharis subulata Ноок. et Ars., etc. Esa especie, perigea de la mitad setentrional del globo, era conocida del hemisferio austral hasta ahora sólo del Estrecho de Magallanes (réase Marc Micheli en DC. Suites au Prodromus, III, 1881, pág. 107).

Van en seguida las determinaciones de las plantas más características de la region andina media que he podido efectuar hasta ahora (').

Plantas de la region andina media

(\%ona de la Leña amarilla, Adesmia pinifolia Grbl.).

\section{A. - Plantas de los lugares secos}

Panunculus peduncularis S.r. - Leñas amarillas, arroyo Tordillo; frecuente.

Berbaris empetrifolia Liм. (Zarzaparrilla).-Mlverjalite hasta Leĩas amarillas, en abundancia; Valle Hermoso.

(1) Los detalles geográficos se encuentran, con pocas excepciones, en el mapa que de la region que tratamos ha publicado mi colega Dr. G. Bodenbender en este mismo Boletin itomo XIII, página 5 y siguientes), pero necesito añadir los siguientes detalles:

$1^{\circ}$ El Arroyo de las Vayas altas es el indicado por G. Bodenberder que desemboca al frente de Los Iolles y recibe por su márgen derecha el Arroyo de los Blancos;

Fo El arroyo que desemboca frente á "Los Pozos » (indicado por G. BoDENBENDER/ es el arroyo Ubilla;

$3^{\circ} \mathrm{El}$ arroyi) llamado por Hodevbexder " $\mathbf{A}$. de las Leñas amarillas » tiene el nombre de " $\mathrm{A}$. de las Agulas amarillas »; el primero es el bajo del Portezuelo ancho;

$4^{\circ}$ En lugar de «Alverjillo» debe ponerse "Alverjalito»;

$5^{\circ}$ Los dos «Cerros de los Molles y de los Baños» pertenecen al mismo grupo que el Cerro de la Hoyada en el mapa de Bodenbender. 
Berberis Grevilleana Gıc. (Crucero, Junquillo en Rodeo Viejo). - Los Molles; baja hasta la Cieneguita.

Hexaptera pinnatifida Gıь. et Hooк.-Alverjalito, Lenas amarillas.

Helianthemum sp. variat floribus purpureis vel albis.Los Molles, Vallas altas; frecuente.

Arenaria serpyllifolia Nico. (?). - Los Molles, hasta Leñas amarillas.

Oxalis platypila GıL. apud Ноок. et Arx. - Alverjalito.

Tropaeolum polyphyllum Car.- Los Molles, Alverjalito, entre Atuel superior y Tordillo; frecueute.

Colletia nana Cuos. - Rio Tordillo, Valle Hermoso.

C. Doniana Cros. - Los Molles, Cajon de Ubilla, Cajon de las Aguas amarillas, Alverjalito. El último lugar es el más elevado del Chacay, que forma aquí arbustos de 1.5 a $2 \mathrm{~m}$. de altura.

Schinus dependens OrT. v. andinus EvgL. - Rodeo Viejo, Los Molles, Alverjalito, Puesto Lbilla.

Cassia Arnottiana Gibl. (Mayu en rio Atuel y Rodeo Viejo). - Entre el Atuel y el Salado, desde la cumbre del Portezuelo ancho hasta la Cieneguita en todas las partes algo húmedas. Es un lindo arbusto de 0.2 hasta $1.5 \mathrm{~m}$. de altura, con hojas algo carnosas de un verde oscuro y con flores grandes color oro (C. Lorentzii Nıenerc.).

Anarthrophyllum rigidum (GILL.) Втн. et Ноок.Porteznelo de la Cieneguita, Los Pozos, Atuel entre Los Arroyos y el Puesto de Ubilla.

Adesmia pinifolia Gict. (Leña amarilla, Colí-mámil).De todas las plantas leñosas de la region tratada, la más característica y la más frecuente. Se extiende del Portezuelo de la Cieneguita hasta Leñas amarillas bajo la cumbre del Portezuelo ancho, formando á reces bosques densos en las pendientes de los Cerros de los Molles y de los Baños, entre Los Pozos y el Arroyo de los Morros, encontrándose tambien 
en abundancia en los valles del rio Tordillo y del Atuel (muy escaso en el Valle Hermoso), cerca del Rodeo Viejo, en el Malal-lué superior, etc. El aspecto de la Leña amarilla es muy característico: de un tronco amarillento salen muchas ramas que toman todas más ó menos la direccion vertical formando un ramaje compacto cubierto de hojas finamente cortadas de un verde claro, imitando los grupos de penachos del Pinus ó más bien de una Larix. ìn el año se cubren las extremidades de los ramos de las masas blanquiscas lanosas de las frutas.

Adesmia trijuga Gıc. var. robusta Ноок. (Choiquemámil). - Portezuelo de la Cieneguita, Los Molles, Alverjalito, Rio Tordillo. Despues de la Leña amarilla es el arbusto alto más frecuente, pero no sube á alturas tan considerables como aquella. Tiene un tronco rojizo, y un ramaje poco denso.

Astragaius unifultus L'Her. (Yerba loca). - Muy comun cerea de los caminos en todas partes.

Lathyrus anomalus Phic. (?).-Leñas amarillas.

L. macropus Gics. (Agua de Nieve). -- En toda la region indicada muy frecuente desde los valles hasta el límite de las plantas leñosas. Linda planta con racimos grandes de flores blanco-azuladas ó blanco-rosadas fragantes. Los animales no comen esta planta, de tal modo que se ha extendido tanto que en algunos puntos, comosucede cerca de Alverjalito, parece ser una planta sembrada.

Acaena (Cardillo). - De este género se observau á lo menos tres especies en el valle del Rio Salado, una de Jas cuales llamada "Cepa-caballos de la Sierra " tiene propiedades estomacales y es muy conocida entre los serranos que la toman haciendo una infusion de la raiz leñosa cortada en pedacitos.

Tetraglochin strictum POEPP. (Margyricarpus alatus GrLL. et Ноок.).- Portezuelo de la Cieneguita, Rio Salado al frente de Alfalfito. 
Gayophytum humile A. Juss. - Los Molles, etc.; frecuente en pendientes arenosas.

Cajophora Kurtzii UnB. et GıL. (Ortiga).-Los Molles, Cajon de las Aguas amarillas. Al Sur hasta la laguna Carilauquen.

Cajophora scandens MEx. et KL. (Ortiga caballuna). Puesto de Ubilla en el Atuel superior, Arroyo de los Molles.

Loasa tricolor Ker. var mendocina Uri. et GiLg.- Valle Hermoso.

Malesherbia solanoides Mex.-Desde el Portezuelo de la Cieneguita y la Laguma Blanca hasta Alverjalito, frecuente. Tambien la he encontrado cerca del Rodeo Viejo pero no más al Sur.

Azorella Gilliesii Ноок.-Desde Alfalfito hasta el Portezuelo ancho, formando en el valle y en las pendientes de los Cerros, céspedes densísimos hemisféricos, que tienen hasta un metro de diámetro y 25 centímetros de altura.

Mulinum spinosum Pers. (Yerba Negra, Chinchimari, Chinchimal en Rodeo Viejo). - Muy comun en todo el valle del Salado desde $\mathrm{A}$ /falfito lıasta Alverjalito.

Phyllactis sp. - Solamente encontrado entre los escombros basálticos del Infierno chico, cerca de la laguna de las Piedras negras.

Baccharis microphylla Ктн. in Н. et B. - Arroyos de los Volles y de Ubilla.

B. subulata (Don Mss.) H. et A.- Los Molles.

Gutierrezia spathulata (Pıı.) m. (Yerba del Buitre).Vayos altas, Rodeo Viejo.

Haplopappus glabratus PhıL.-Alverjalito; frecuente cerca del arroyo.

Senecio albicaulis $\mathbf{H}$. et $\mathrm{A}$. - Comun en las regiones bajas del valle del Salado.

S'. holophyllus Revr. - Los Molles, Alverjalito; frecuente. 
S. salsus Griseb. - Alfalfalito.

Chuquiraga oppositifolia Gır.. et Dor (Retamillo).Iuy frecuente en la parte inferior de las pendientes hasta Leñas amarillas.

Doniophytum anomalum (Dos) m. (Chuquiraga anomala Dos, Doniophytum andicolum WeDr.) - Los Molles. Lo he observado de San Rafael y Los Molles en el Norte hasta Agua Botada (Rio Grande) en el Sur.

Mutisia retrorsa Cav. (M. runcinata W.).-- Los Molles y abaju hasta la Cieueguita ; raro.

Nassauvia (Stronggloma) axillaris (DC.) Втн. et Ноок. - Desde el Portezuelo de la Cieneguita liasta Los Molles y más arriba (hasta Los Morros) muy frecuente, formando, cubierto de sus llores blancas, en las pendientes de los Cèrros grupos casi coralineos.

Achyrophorus andinus DC. (?).-Los Molles, en lugares algo liúmedos.

Gentiana. - Pantanos arriba de Alverjalitos, Atuel al pié del Cerro Sosneado.

Trechonaetes laciniata Miens. - Leñas amarillas.

Calceolaria plantaginea S. - Rocas húmedas, orillas de los arroyos de toda la region indicada.

Melosperma andicola Bтн. - Comun en lugares arenosos de todo el valle, hasta Alverjalito.

Mimulus luteus I. - En los mismos lugares pero más frecuente que la Calceolaria.

Schizanthus. - Portezuelo de la Cieneguita, Los Molles, Valle Hermoso, entre Atuel superior y Tordillo. Parece se cría aquí una especie en dos formas: en los valles se presenta como planta más alta, de llores color rosa, y en los sitios más elevados aparece mis baja con llores purpúreas.

Argylia Bustillosii Prat.-Muy frecuente en las faldas de todo el valle del Salado, tapizando muchas veces grandes espacios con su ramaje verde-claro y sus grandes llores amarillas. En el Sur lo he encontrado hasta Codi-hué (Rio Igrio). 
Dipyrena glaberrima GıL. et Ноок. - Uno de los ar bustos más frecuentes del valle: sube hasta cerca del límite de la Leña amarilla.

Verbena juniperina LaG. Entre Alverjalito y Leñas amarillas.

V. microph pylla Ктн. in H. et B.-Atuel superior, Leñas amarillas.

V. spathulata GiLl. et Hook.-Los Molles hasta Alverjalito; Cerro de los Molles, frecuente. Tiene flores blancas, azules ó rosadas.

Oxyteca dendroidea Nutt. - Los Molles, colinas arenosas.

Urtica sp. - Los Molles, los Morros.

Sisyrrhynchium flexuosum Pнıь. - Leñas amarillas. Rhodolirion montanum PHIL._Los Molles, Alverjalito, Valle Hermoso. Muy frecuente en los dos primeros lugares, guarneciendo con sus grandes flores purpureas las pendientes bajas.

Carex atropicta Steud. - Valle Hermoso ; raro.

Bromus macranthus MEY. - Alverjalito, Rio Tordillo.

Dyeuxia andina PHı. - Los Molles, Alverjalito, Valle Hermoso, Rio Tordillo.

Festuca acanthophylla Desv. - Alverjalito, Valle Hermoso, Rodeo Viejo.

Poa annua L.-Pucsto de Ubilla, Atuel superior; en abundancia.

Slipa Neaei Nees.-- Los Molles, frecuente; no lo he observado más arriba.

St. (sp. nov. grege Pappophorearum). - Desde la embocadura del Salado hasta los Morros; entre este lugar y Los Pozos es el pasto principal.

Ephedra americana W.- Alfalfalito hasta Los Morros, muy frecuente; tiene frutas coloradas, blancas y rosadas, comestibles. 
B. - Plantas de las orillas del rio, arroyos, ciénegas y pantanos

Ranunculus Cymbalaria Pursch. - Muy frecuente en los bordes de los arroyos.

Senccio Iualtata BERT. - Forma grandes grupos en el arroyo de la Laguna de las Piedras negras.

Baccharis subulata (Dox mss.) H. et A. - Barro sulfuroso de los baños de Los Molles.

Solidago linearifolia DC. - Se encuentra casi siempre cerca del agua.

Anagallis alternifolia CAv. - Pantano de la Cieneguita, cerea de Los Morros; al Sur la he observado hasta Copa-hué, al poniente de Norquin.

Glaux mucronata PurL. - Orillas húmedas arenosas del Atuel, al pié del Cerro Sosneado.

Plantago macrostachys Dcne. - Pantano de la Cieneguita.

Quinchamalium sp.-Orillas del Salado al frente de Los Molles.

Juncus Mandoni Buch. (J. depauperatus Phi. ex specimine autor.). - Pantano de la Cieneguita; arroyitos cerca de la cumbre del Cerro de los Molles; frecuente.

Triglochin maritima L. - En el barro sulfuroso de los Baños de los Molles, muy frecuente. Hasta ahora, en el hemisferio austral era conocida sólo del Estrecho de Magallanes.

Scirpus riparius Prese., forma inflorescentia contractil . - Laguna de las Piedras negras, ciénega cerca de Los Pozos, formando grandes matorrales.

Festuca acanthophylla Desv.- Alverjalito, cubre grandes espacios de terreno pantanoso.

Gynerium argenteum NeEs. - En todas partes cerca del rio.

Hordeum comosum Prest. - La Cieneguita, Los Molles, 
Rodeo Viejo, Portezuelo ancho. Las espigas muy frágiles son de un color negro-purpúreo, ó negro-azulado.

Distichlis thalassica (Ктн. in H. et B.) Desv.- Baños t!e Los Molles.

Plantas de los terrados del Rio Salado, cerca de los Molles

Asteriscium chilense Cr. et Schudd. - Planta robusta $(0.25 \mathrm{~m}$. de altura) coriácea, de un verde amarillento ; alggunos grupos aislados.

Baccharis subulata (Don mss.) Hook. et A., var. B. Una forma postrada pero mostrando todos los caracteres de la forma típica erecta.

Sporolobus deserticola PHIL. - Linda gramínea muy delicada, frecuente entre las piedras redondas de la orilla del rio, encontrándose tambien cerca de la laguna de las Piedras negras.

3) Region andina superior

(Zona entre el límite superior de la Leña amarilla y la línea inferior de la nieve perpétua;

Un poco más arriba del arroyo Alverjalito, la vegetacion cambia considerablemente; los únicos arbustos son: Adesmia pinifolia GuL., Berberis empetrifolia Ian. y Argylia Bustillosii Phı., los dos últimos cubriendo largos espacios de las faldas. Aqui aparecen especies de Acaena, (Cepa-caballo de la sierra), Laiyhrus anomalus Phr., Oxytropis, Epilobium (2 ó 3 especies), Carex, Arenaria, Plantago, Ranunculus, Verbena juniperina G. et H., 
$V$. microphylla Kтн. in H. et B., un número erecido de Compuestas, Hexaptera pinnatifida GrLl. et Ноок., Sisyrrhynchium flexuosum PHı., y los pantanos contienen grandes grupos de un Senecio alto, semejante al S. Hualtata Bent., al lado de la cruel Festuca acanthophylla Desv.

En fin, hemos pasado ya la region de las plantas leñosas y subiendo al Porteznelo ancho, que divide las aguas que corren al Este al valle del Salado, de los arroyos que bajan al Poniente al Valle Hermoso, nos saluda una veretacion baja, pero muy interesante y generalmente con lindas flores grandes. Una excepcion en este sentido hace la planta principal de los terrenos pantanosos, la Ochychloë andiná Pнı., una Juncácea rígida con hojas algo punteadas, que forma grupos densísimos hemisféricos hasta de $1 \mathrm{~m}$. de diámetro y 0.6 $m$. de altura. Entre las demás plantas, son de notarse una Brodiaea con flores estriadas de blanco y negro (B. Poeppiggiana m.), Chamelum Bodenbenderi m. (linda plantita semejante á un Crocus con flores blanco-rosadas), Cajophora pulchella Urb. et Gilg., que se cría cerca de la nieve, la curiosa Barneoudia chilensis GaY, con flores morenas que aparecen antes que las hojas, atravesaudo la nieve, como lo hacen las flores de Soldanella en los Alpes, y el Phleum alpinum L., gramínea conocida de Europa, Asia y de las dos partes de América.

Algo diversa de la flora formada por las plantas arriba mencionadas, que se crían en un suelo húmedo ó turboso, reunidas de vez en cuando en pequeñas bandas verdes, es el aspecto de la vegetacion de los escombros, como lo he podido estudiar en las cumbres de los Cerros de los Molles y de los Baños. Aquí se encuentran los individuos de las plantas aisladas, apenas visibles entre las piedras y algunas especies disimulan aún más su presencia á causa de la semejanza de sus colores con las rocas, (por ejemplo, Barneoudia chilensis Gay., las especies de Tiola del grupo Rosulatae, Calandri- 
nia picta Gill., Pozoa hydrocotylifolia Field et Gardn., Chaetanthera villosa GILL.). Las plantas más características de esta region sou : Loasa petrophila Urb. et GiLL., con lindas flores blancas y hojas finamente recortadas, la Leuceria Contrayerba m. y el Culcitium Poeppigii DC. La Leuceria llamada "Contrayerba" es una plantita baja con una roseta de hojas algo gruesas, y grandes flores porceláneas, purpureas por fuera, blancas adentro; los serranos la buscan y usan su raíz para dar fragancia al tabaco. El Culcitium Poeppigii, la Vira-vira de la Cordillera de Mendoza, es una planta robusta, gris, con cabezuelas amarillas y á la que los serranos atribuyen virtudes contra la tos convulsa.

\section{Plantas de la region andina superior}

(Zona entre el límite superior de la Leña amarilla y el inferior de la nieve perpétua)

Barneoudia chilensis Gay var. major (Phr.) m. Portezuelo ancho, Cerro de los Molles.

Ranunculus peduncularis S. var. minor IVEDD. Portezuelo ancho, Rio Tordillo superior.

Hexaptera pinnatifida GıL. et Ноок. - Cerro de los Molles.

Violae sp. (Rosulatae). - Cerro de los Molles, Paso entre Atuel superior y Tordillo.

Cerastium. - De este género se observan á lo menos dos especies en la region superior, pero hasta ahora no he podido determinarlas.

Calandrinia affinis GıL. - Portezuelo ancho, Valle Hermoso.

C: dianthoides PHu. - Vayas altas, Cerro de los Molles. 
C. picta Gilt. - Cerros cerca de Alverjalito (frecuente), Paso entre Atuel superior y Tordillo.

Oxalis compacta Gitu. - Portezuelo ancho.

Colletia nana GLos. - Portezuelo aucho; los troncos, medio subterráneos, son gruesos hasta $3 \mathrm{~cm}$. y de un largo hasta $1.5 \mathrm{dm}$. y las ramas no tienen más de 1 á $2 \mathrm{~cm}$. de largo. Cubre como una alfombra densa, pero muy espinosa, las piedras.

Lathyrus anomalus PhiL. (?). - Cerro de los Molles, á la orilla de un arroyito bajo la Cumbre.

Cajophora pulchella Uri. et Gı́. - Portezuelo ancho, cerca de la nieve.

Loasa petrophila Urb. et GrLG. - Cerro de los Molles. Recogida por primera vez en el Cajon de Trolope, al Oeste de Norquin.

Larretia acaulis Gibl. "et Ноок. - Portezuelo ancho, Paso entre Atuel superior y Tordillo; la raíz es muy aromática.

Mulinum spinosum Pers. $f$. humilis fructibus brunneis. - Cerro de los Molles; planta compacta de sólo 0.5 á $1 \mathrm{dm}$. de altura y con frutas más grandes que las de la forma típica de la Yerba negrra.

Pozoa hydrocotylifolia Fielo. et Gardx. - Cerro de los Molles (escombros de la Cumbre), Cerro de los Baños, Paso entre Atuel superior y Tordillo.

Sanicula macrorrhiza Colla. - Vayas altas, Portezuelo ancho.

Boopis sanjuanina Hierox. - Cerro de los Molles; escasa.

Culcitium Poeppigii DC. (Vira-vira). - Faldas superiores del Cerro de los Molles y de los cerros vecinos; frecuente; encuéntrase algunas veces en los valles, traída por la corriente, como por ejemplo, en el del Papagayo. Esta. planta es la "Vira-vira " de Mendoza y se usa en forma de arabe para combatir la tos convulsa en los niños (Gnapha- 
lium luteo-album L. y Senecio Vira-vira Hieron. tienen aquí el mismo nombre y se les atribuye las mismas ó semejautes propiedades; véase Hıronymus, Plantae diaphoricae Florae Argentinae, pág. 150, 160).

Caloptilium Lagascae Ноок. et Ans. - - Cerro de los IIolles; escasa.

Chaetanthera villosa GiLt, (Carmelita formosa Gar). - Cerros cerca de Alverjalito; rara.

Leuceria Contrayerba m. (Contrayerba). - Cerro de los Baños (recogida antes en la sierra de Loncoche). Los arrieros y vaqueanos usan la raíz de esta planta para dar fragancia al tabaco y dicen que posee tambien propiedades estomacales.

Nassauvia axillaris (DC.) Втн. et Ноок. - Cerro de los Molles, Cerro de los Baños.

N. glomerata (Gill.) Wedd. - Paso entre Atuel superior y Tordillo; escasa.

Perezia carthamoides (Dov) Hook. et Ans. - Vayas altas; linda planta con grandes flores color crema que tienen una fragancia parecida á la Vainilla.

P. (Clarionea) pilifera (Dos) m. - Portezuelo aucho.

Trechonaetes laciniata Miers. - Portezuelo ancho.

Verbena erinacea GiLL. et Ноок. - Vayas altas.

Plantago uncialis Dcne. - Portezuelo ancho.

Rumev magellanicus CAMPD. - Paso entre Atuel superior y Tordillo.

Chamelum Bodenbenderi m. - Portezuelo ancho.

Sisyrrhynchium flexuosum PніL. - Vayas altas, Cerro de los Molles, Portezuelo ancho.

S. ex affinitate $S$. floribundi PHIL. flor. purpureis.Portezuelo ancho, Cerro de los Molles.

Brodiaea Poeppigiana (GAY) m. - Portezuelo ancho.

Juncus Mandoni Buch. - Cerro de los Molles.

Oxychloë andina PнгL. - Frecuente en la region superior, formando grupos densos cerca de los arroyos. (Cerro 
de los Molles, Vayas altas, Portezuelo ancho, entre Atuel y Tordillo).

Deyeuxia andina Phis. f. humilis m. - Portezuelo ancho.

Phleum alpinum L. - Portezuelo ancho.

En suma, por la descripcion que acabo de hacer se desprende que la flora de esta parte de la Cordillera no es tan rica como la de la parte entre el Neuquen y el Limay, y las investigaciones que se hagan más tarde probarán cada vez más el hecho que desde ya se observa, de que la flora de la falda oriental de los Andes no es sinó una vegetacion empobrecida con respecto á la de la falda chilena, con muy pocos elementos propios, fenómeno que debe atribuirse en primer lugar á las diferencias climatológicas de ambas vertientes y que tiene una completa analogía con la diferencia existente entre las floras de los dos lados de la Sierra Nevada en NorteAmérica, donde contrasta la aridez y escasez de plantas de la falda oriental con la rica y variada vegetacion que adorna la pendiente del lado de California.

Córdoba, Junio de 1893. 


\title{
APENDICE ]
}

\author{
LITERATURA
}

1. Ноокеr, W. J. On the Genus Colliguaya of Molina, with an account'of three new species. - Hooker's Botanical Miscellany. Vol. 1, 1830, p. 138-143, tabb. XXXIX-XL.

- On the species of the Genus Colletia, of the Natural Order Rhamneae, discovered by Dr. Gillies in South America. - Ibid. p. 150-159, tabb. XLIIIXLV.

- On the species of the Genus Verbena, and some nearly allied Genera, found by Dr. Gillies in the extratropical parts of South America. — Ibid. p. 159-173, tabb. XLVI-XIIX.

- On the plants of the Natural Order Umbelliferae, dctected by Dr. Gillies in the extratropical parts of South America. - Ibid. p. 343-335, tabb. LXIIILXVII.

- On a new Genus of the Natural Order Cruciferae, from the Audes of Chile and Mendoza. - Ibid. p. 349-352, tabb. LXXII-LXXIV.

- Descriptions of varions plants from Dr. Gillies' Cordilleran Collections. Ibid.
a) Mutisia; p. 7-12, tabb. IV-IX.
b) Poinciana; p. 129-131, tab. XxxIV.
c) Atropa (Salpichroa); p. 135, tab. XXXVII.
d) Jaborosa; p. 347-348, tab. LXXI. 
Ноoкer, W. J. and G. A. Walier Arnott. Contributions towards a Flora of South America and the Islands of the Pacific. I. Extratropical South America; en:

a) Bot. Miscellany III, 1838, p. 129-211, 302367

b) Companion to the Rotanical Magazine I, 1835, p. 29-38, 102-111, 234-244; II, 1836, p. 41-52, 250-254;

c) The Journal of Botany I, 1834, p. 276-296, tab. CXXXVII; III, 1841, p. 19-47, 310-348.

2. Philippi, R. A. Sertum mendozinum. Catálogo de las plantas recogidas cerca de Mendoza y en el camino entre esta y Chile por el Portezuelo del Portillo, por D. Wenceslao Diaz, en los años de 1860 y 1861.-Anales de la Universidad de Chile, XXI, Sautiago, 1862, p. 389-407.

- Sertum mendozinum alterum, ó sea, catálogo de las plantas recogidas cerca de Mendoza y en los caminos que conducen de Chile á esa ciudad. Ibid. XXXIV, 1870, p. 159-212.

3. Cesati, V. DE. Illustrazione di alcune piante raccolte dal Sr. Prof. Strobel sul versante orientale delle Ande Chilene dal Passo del Planchon, sino à Mendoza, attraverso la Pampa del Sud.-Atti Reale Accad. di science fisiche e matemat. V, Napoli, 1871, p. 1-22, tab. III.

4. Echegaray, S. Determinacion de plantas sanjuaninas.Boletin de la Academia Nacional de Ciencias exactas, II, Córdoba, 1875, p. 341-353.

Hieronymus, J. Sertum sanjuaninum ó descripciones de plantas fanerógamas y criptógamas vasculares recolectadas por el Dr. D. S. Echegaray en la Provincia de San Juan. Jbid. IV. 1881-1882, p. 1-73.

5. P. Lorextz y G. Niederceix en: Informe oficial de la Comision científica... de la Expedicion al Rio 
Negro (Patagonia) bajo las órdenes del general D. Julio A. Roca, Buenos Aires, 1881, Botánica, p. 171-296, con 12 láminas.

6. BaLL, J. Further Contributions to the Flora of Patagonia.

- The Journal of the Linnean Society, Botany vol. XXVII, 1891, p. 471-500.

7. Kurtz, F. Informe preliminar de un viaje botánico etc. en las provincias de Córdoba, San Luis y Mendoza hasta la frontera de Chile, en los meses de Diciemhre de 1885 á Febrero de 1886. - Boletin de la Academia Nacional de Ciencias exactas, IX, Córdoba, 1886, p. 349-370.

8. Philippi, F. Catalogus plantarum vascularium chilensium adhuc descriptarum. Santiago de Chile, 1881.

\section{APÉNDICE II}

NOTAS SOBRE ALGUNAS PLANTAS DE LA CORDILLERA MENDOGINA

Leuceria Contrayerba nov. spec.

Leuceria Lagasca, Benthas et Ноокеr, Gen. Plant., II, p. 499.

Planta perennis, humilis, acaulis. Rhizoma simplex, v. 2 $-\infty$ ceps, lignosum, $6-10 \mathrm{~cm}$. longum, 0,5-0,8 cm. crassum, sordide fuscum, cortice demum irregulariter fissa obtectum. Folia basalia, rosulata, petiolata, crassa, subcoriacea, bullata, orbicularia, oblonga $v$. rhomboïdea, basi truncata v. breviter in petiolum attenuata, 1,5-3 cm. longa, 1,5-2,2 cm. lata, margine irregulariter eroso-dentata $\mathrm{v}$. crenata, supra floccosolanata, subtus dense albo-tomentosa; petiolis $1-2,5 \mathrm{~cm}$. longis, basi vaginantibus, subtomentosis. Capitula homoga- 
ma, radiatiformia, majuscula, ad 5 cm. lata, solitaria, pedicellata; pedicellis $3-7,5 \mathrm{~cm}$. longis, purpurascentibus, parce lloccosis. Flores radii uniseriati intus albi extus purpurascentes, parce lanati, glandulis flavis minutis obsiti; flores disci llavi, pappus albus.

Involucrum campanulatum, bracteis subtriseriatis, imbricatis, herbaceis, acuminatis, exterioribus minoribus (ad 0,5 cm. longis), interioribus maximis (ad $1, \overline{\mathrm{cm}} \mathrm{cm}$. longis), apice purpurascentibus, margine scariosis, dentibus perpancis minutis munitis, extus grlandulis flavis minimis more corollarum radii onustis, omnibus lana alba floccose obtectis. Receptaculum planum, epaleatum, favosum, areolis marginibus scariosis apice leviter laceratis cinctis. Corollae bilabiatae; llores radii labio exteriore 3 -dentato, elongato, liguliformi (ad 1,2 cm. longo), interiore 2-partito, segmentis longis, revolutis; disci flores labiss subaequilongis, exteriore breviter 3 -dentato, interiore profundius 2-partito, revoluto, fauce extus pilis paucis minutis adspersi. Antherae radii papillosae, acuminatae, basi sagittatae, caudis papillosis, filamentis brevibus, non dilatatis nec !papillosis; antherae disci fauci insertae glabrae, acuminatae, basi longe caudatae, caudibus barbatis, filamentis basi dilatatis papillosis. Styli radii claviformes, ramis brevissimis, erectis, rotundatis, minute papillosis; styli disci filiformes ramis brevissimis, erectis, rotundatis, capitulum quasi formantibus (more Chaetantherae villosae GrL., Oriastri pusilli Poepp. et EnnL., Eganiae acerosae Revy). Achaenia mmatura obconica, angulis 4 prominulis, dense albo-papillosa, ad $3 \mathrm{~mm}$. longa. Pappi setae radii 2 -seriatae, disci 1 seriatae (?), scabro-serrulatae.

Species nullae aliae generis Leuceriae mihi cognitae affinis habitum Trichoclines aemulans, a qua generice characteribus supra indicatis et insuper colore et distributione nervorum florum radii diversa, ad sectionem a BExтнамио l. c.: "capitulis majoribus in scapo aphyllo v. caule simplici solitariis » definitam pertinet. 
Crescit inter lapides regionis superioris-supra limitem plantarum lignosarum-Andium provinciac Mendozae Reipublicae Argentinae: Portezuelo de Loncoche (F. Kurtz, Herb. argentin. No 5978; 7, Il (1888); Cerro de los Baños, Valle del Rio Salado superior (F. Kurtz 1. c. No 7659 ; 11, II, 1893).

"Contrayerba" ab inq̧uilinis appellatur.

Planta tota, praecipue radix quidem, gratam fragrantiam exhalat, quam ob rem ab"incolis Andium aegre quaeritur; eam ad tabaci odorem meliorandum utuntur. Praeterea infusio theiformis contra stomachi dolores applicatur.

\section{Verbena aphylla Gru.. ет Ноок.}

Verbena aphylla GiLL. et Ноoк. in W. J. Hooken's Bot. Miscellany I, 1830, p. 161.

Haec species in ramis junioribus, saepe flexuosis, apicem versus pilis albis brevibus obsitis folia minuta (ab illi. GıLles et НоокеR non observata) exhibet opposita, breviter (petiolum ad $1 \mathrm{~mm}$. longum) lateque petiolata, oblonga, 1-4 $\mathrm{mm}$. longa, 0,5-1,2 mm. lata, obtusa $\mathrm{v}$. acutiuscula, crassiuscula, caducissima.

Spicae florum pulchre lilacinorum terminales $\mathbf{v}$. laterales.

Verbena aphylla G. et H. inter plantas praedominantes formationis subandinae provinciae Mendozae arbustus (ad $1 \mathrm{~m}$. altus) insignis, fruticeta diffusa formans.

Observavi inter torrentes Arroyo Papagayos et Arroyo de la Faja (F. Kurtz, Herb. argentin. $\mathbf{X}^{\circ} 7422 ; 27$, XII, 1892). 
C'hamelum Bodenbenderi nor. spec.

Chamelum R. A. Philippl; Benthas et Hooker Gen. plant.. III, p. 700.

Planta omnino glabra. Rhizoma fuscum oblique adscendens, nodoso-articulatum, ramosum, 1,כ̌-3) mm. crassum, subtus radices fuscas, carnosulas, ad $4,5 \mathrm{~cm}$. longas emittens. in apicibus ramorum folia floresque gerens. Folia fasciculorum exteriora vaginiformia, viridi-fuscescentia, 2-6 cm. longa, 4-6 mm. lata, membranacea, striata, apiculata; interiora lineari-filiformia, rígida, glaucescenti-viridia, striata, $8-13 \mathrm{~cm}$. longa, 1,5-2 mm. lata, compressiuscula, mucronato-

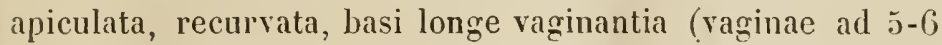
$\mathrm{cm}$. longae), flores superantia, juniora apicem versus pilis paucis brevibus albis obsita. Scapus ex axilla unius foliorum exteriorum vaginiformium ortus validus, $1,5-4 \mathrm{~cm}$. altus, teres, saepe foliis basalibus complete obtectus, glaber. Spathae terminales, 2 v. rarins 3-florae; bracteae (et prophylla) membranaceae, apice apiculatae $\mathrm{v}$. interdum laminuligerae. striatae, viridi-purpurascentes, $2,5-4 \mathrm{~cm}$. longae, $3,5-8 \mathrm{~mm}$. latae, superiores saepe apice plus minus laceratae. Flores breviter pedicellati (pedicelli circa $3 \mathrm{~mm}$. longi); perianthium infundibuliforme, ad $6,5 \mathrm{~cm}$. longum; tubus $18-20 \mathrm{~mm}$. longus, tenuis, ad basin vix $1,5 \mathrm{~mm}$. latus, intus lutescens; lobi lanceolati, obtusiusculi, erecto-patentes, subaequales, albae, nervis purpureis. Antherae $6, \tilde{5} \mathrm{~mm}$. longae, basi profunde sagittatae, tubum filamentorum aequantes. Ovarium oblongum, circa $5 \mathrm{~mm}$. longum, $2 \mathrm{~mm}$. latum; stylus filiformis, antheris longior, ramis brevissimis (2 mm.), leviter apertis, apice apiculum gerentibus.

Crescit in jugo "Portezuelo ancho" dicto Andium Mendozae inter vallem fluminis "Rio Salado» et vallem "Valle 
Hermoso " appellatum (F. Kurtz, Herb. argentin. $N^{\circ} 5835$; 15, I, 1888, et No $7180 ; 1$, II, 1892$)$.

A Chamelo luteo R. A. Phucıppi differt Ch. Bodenbenderi mihi statura duplo majori, glabritie, foliis mucronatoacuminatis, floribus 2-v. 3-plo majoribus, rosaceo-albidis. Ch. andinum (R.A. Рнгь.) Ноок. fil. floribus luteis gaudet et ex descriptione PhicıppI variis aliis notis a specie nostra diversum.

Pulchellam hanc plantám amico itinerumque socio carissimo, in Universitate cordobensi collegae, Dr. G. BoDENBeNDER dedicavi. 







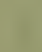
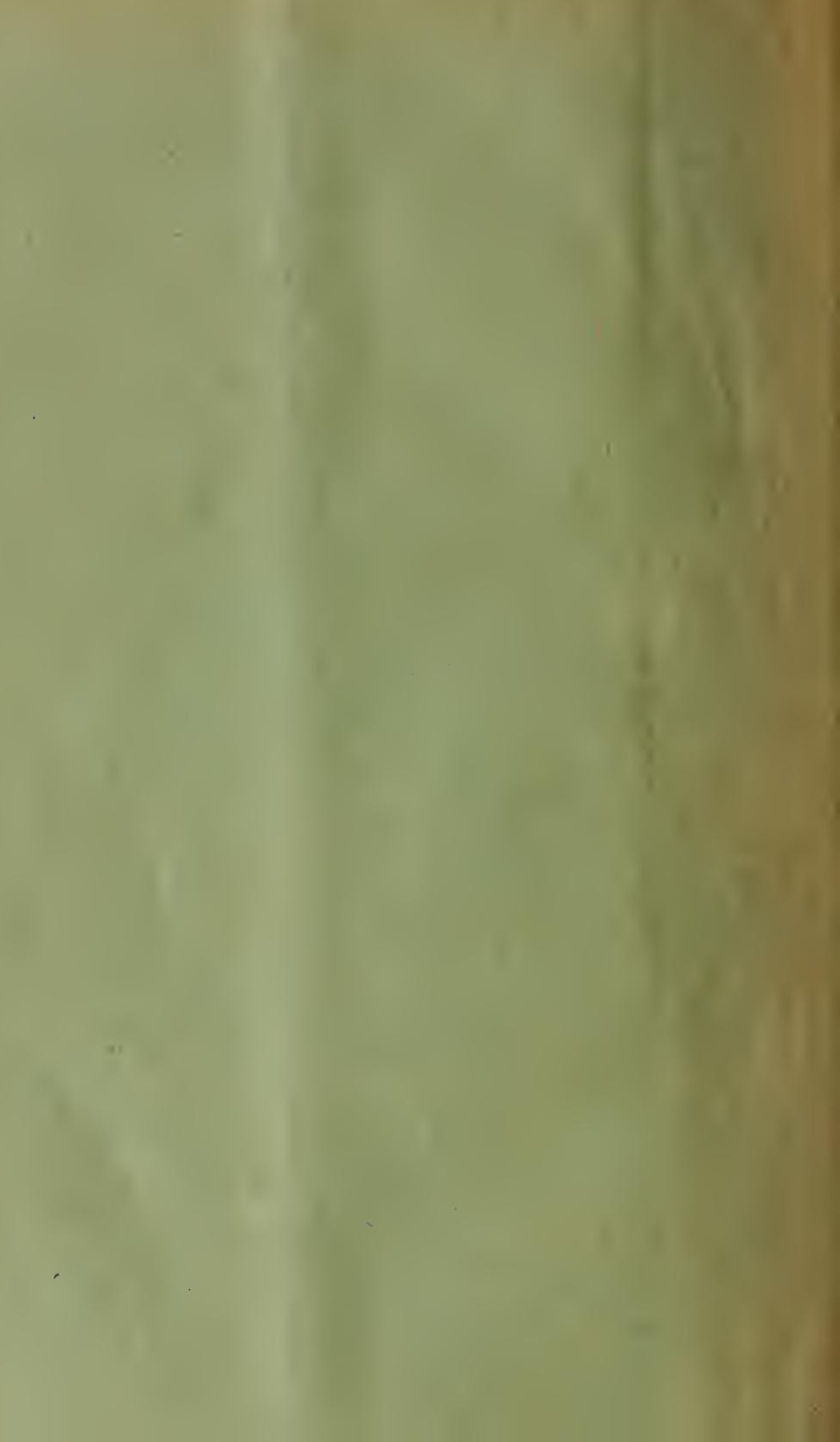
OK 257. Kew York Botanical Garden Library

Kurtz, Federico/Dos viajes botanicos al

3 5185001024924 
